\title{
Two Decades of KASCADE and KASCADE-Grande Measurements: Some Achievements
}

\author{
A. Haungs $^{* 1}$, W.D. Apel ${ }^{1}$, J.C. Arteaga-Velázquez ${ }^{2}$, K. Bekk ${ }^{1}$, M. Bertaina ${ }^{3}$, \\ J. Blümer ${ }^{1,4}$, H. Bozdog ${ }^{1}$, I.M. Brancus ${ }^{5}$, E. Cantoni ${ }^{3,6}$, A. Chiavassa $^{3}$, \\ F. Cossavella ${ }^{4}$, K. Daumiller ${ }^{1}$, V. de Souza ${ }^{7}$, F. Di Pierro ${ }^{3}$, P. Doll ${ }^{1}$, R. Engel ${ }^{1}$, \\ D. Fuhrmann ${ }^{8}$, A. Gherghel-Lascu ${ }^{5}$, H.J. Gils ${ }^{1}$, R. Glasstetter ${ }^{8}$, C. Grupen ${ }^{9}$, \\ D. Heck ${ }^{1}$, J.R. Hörandel ${ }^{10}$, D. Huber ${ }^{4}$, T. Huege ${ }^{1}$, K.-H. Kampert ${ }^{8}$, D. Kang ${ }^{4}$, \\ H.O. Klages ${ }^{1}$, K. Link ${ }^{4}$, P. Łuczak ${ }^{11}$, H.J. Mathes ${ }^{1}$, H.J. Mayer ${ }^{1}$, J. Milke ${ }^{1}$, B. Mitrica ${ }^{5}$, \\ C. Morello ${ }^{6}$, J. Oehlschläger ${ }^{1}$, S. Ostapchenko ${ }^{12}$, N. Palmieri ${ }^{4}$, T. Pierog ${ }^{1}$, H. Rebel ${ }^{1}$, \\ M. Roth ${ }^{1}$, H. Schieler ${ }^{1}$, S. Schoo ${ }^{1}$, F.G. Schröder ${ }^{1}$, O. Sima ${ }^{13}$, G. Toma ${ }^{5}$, \\ G.C. Trinchero' ${ }^{6}$, H. Ulrich ${ }^{1}$, A. Weindl ${ }^{1}$, J. Wochele ${ }^{1}$, J. Zabierowski ${ }^{11}$ - \\ KASCADE-Grande Collaboration \\ ${ }^{1}$ Institut für Kernphysik, KIT - Karlsruhe Institute of Technology, Germany \\ ${ }^{2}$ Universidad Michoacana, Inst. Física y Matemáticas, Morelia, Mexico \\ ${ }^{3}$ Dipartimento di Fisica, Università degli Studi di Torino, Italy \\ ${ }^{4}$ Institut für Experimentelle Kernphysik, KIT - Karlsruhe Institute of Technology, Germany \\ ${ }^{5}$ Horia Hulubei National Institute of Physics and Nuclear Engineering, Bucharest, Romania \\ ${ }^{6}$ Osservatorio Astrofisico di Torino, INAF Torino, Italy \\ ${ }^{7}$ Universidade São Paulo, Instituto de Física de São Carlos, Brasil \\ ${ }^{8}$ Fachbereich Physik, Universität Wuppertal, Germany \\ ${ }^{9}$ Department of Physics, Siegen University, Germany \\ ${ }^{10}$ Dept. of Astrophysics, Radboud University Nijmegen, The Netherlands \\ ${ }^{11}$ National Centre for Nuclear Research, Department of Astrophysics, Lodz, Poland \\ ${ }^{12}$ Frankfurt Institute for Advanced Studies (FIAS), Frankfurt am Main, Germany \\ ${ }^{13}$ Department of Physics, University of Bucharest, Bucharest, Romania \\ E-mail: Andreas. Haungs@kit.edu
}

\begin{abstract}
At a few PeV the energy spectrum of high-energy cosmic rays exhibits a sudden change of the spectral index, usually known as the knee of the spectrum. The origin of this knee is seen as key for the understanding of galactic cosmic rays. KASCADE-Grande investigated the elemental composition of cosmic rays in the energy region of the knee in detail with a multi-detector installation (including LOPES for measuring the radio emission of air showers). The history of the KASCADE installation, the most important results, as well as some implications of these results for the understanding of cosmic ray physics will be summarized in this contribution. In addition, lessons learnt from KASCADE for future experiments will be discussed.
\end{abstract}

The 34th International Cosmic Ray Conference,

30 July- 6 August, 2015

The Hague, The Netherlands

\footnotetext{
* Speaker.
} 


\section{KASCADE-Grande}

The Extensive Air Shower (EAS) experiment and facility KASCADE-Grande stopped finally the active data acquisition end of 2012 and is meanwhile fully decommissioned. The collaboration, however, continues the detailed analysis of nearly 20 years of high-quality air-shower data. Moreover, with KCDC, the KASCADE Cosmic-ray Data Center, we provide to the public the edited data via a customized web page.

The multi-detector experiment KASCADE [1] (located at $49.1^{\circ} \mathrm{n}, 8.4^{\circ} \mathrm{e}, 110 \mathrm{ma}$ a.s.1.) started operation in 1993 and was extended to KASCADE-Grande in 2003. Main parts of the facility were the Grande [2] array spread over an area of $700 \times 700 \mathrm{~m}^{2}$, the original KASCADE array covering $200 \times 200 \mathrm{~m}^{2}$ with unshielded and shielded detectors, a large-size hadron calorimeter, and additional muon tracking devices. The radio antenna field LOPES [3] and the microwave experiment CROME [4] were also important components of the experimental set-up of KASCADE-Grande.

The main goal of the measurements was the estimation of energy and mass of the primary particles. The analysis is based on the combined investigation of the charged particle, the electron, and the muon components measured by the detector arrays of Grande and KASCADE. The general ansatz of the KASCADE and KASCADE-Grande experiments is the determination of the chemical composition in the primary energy range $10^{14}-10^{18} \mathrm{eV}$ by reconstructing individual mass group spectra. Structures observed in these individual spectra provide strong constraints to astrophysical models of origin and propagation of high-energy cosmic rays.

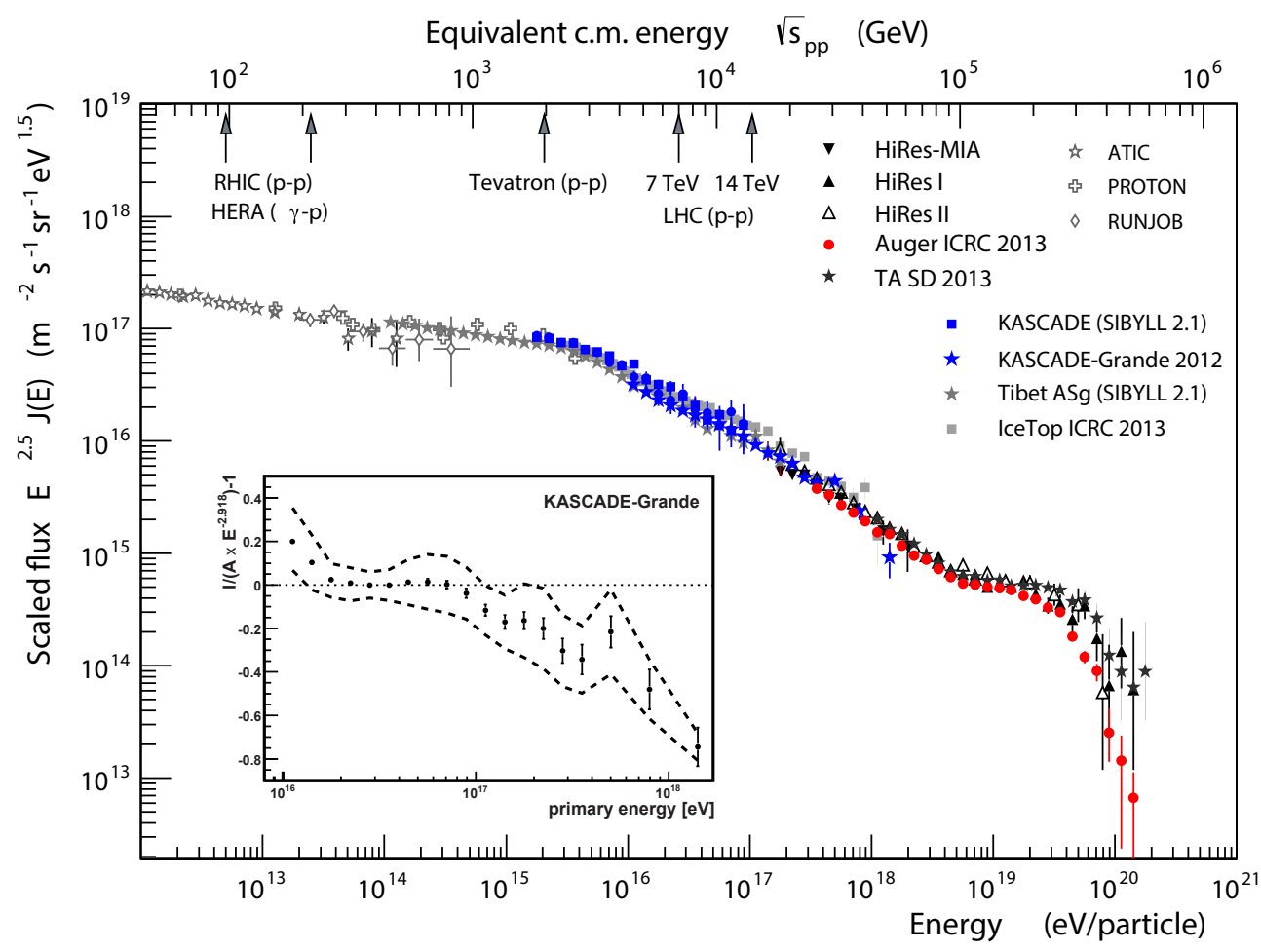

Figure 1: The all-particle energy spectrum obtained with KASCADE and KASCADE-Grande (based on the QGSJet-II model and unfolded, i.e. corrected for the reconstruction uncertainties). The inlet displays the spectrum of KASCADE-Grande [5] in a way to better see the spectral features. 

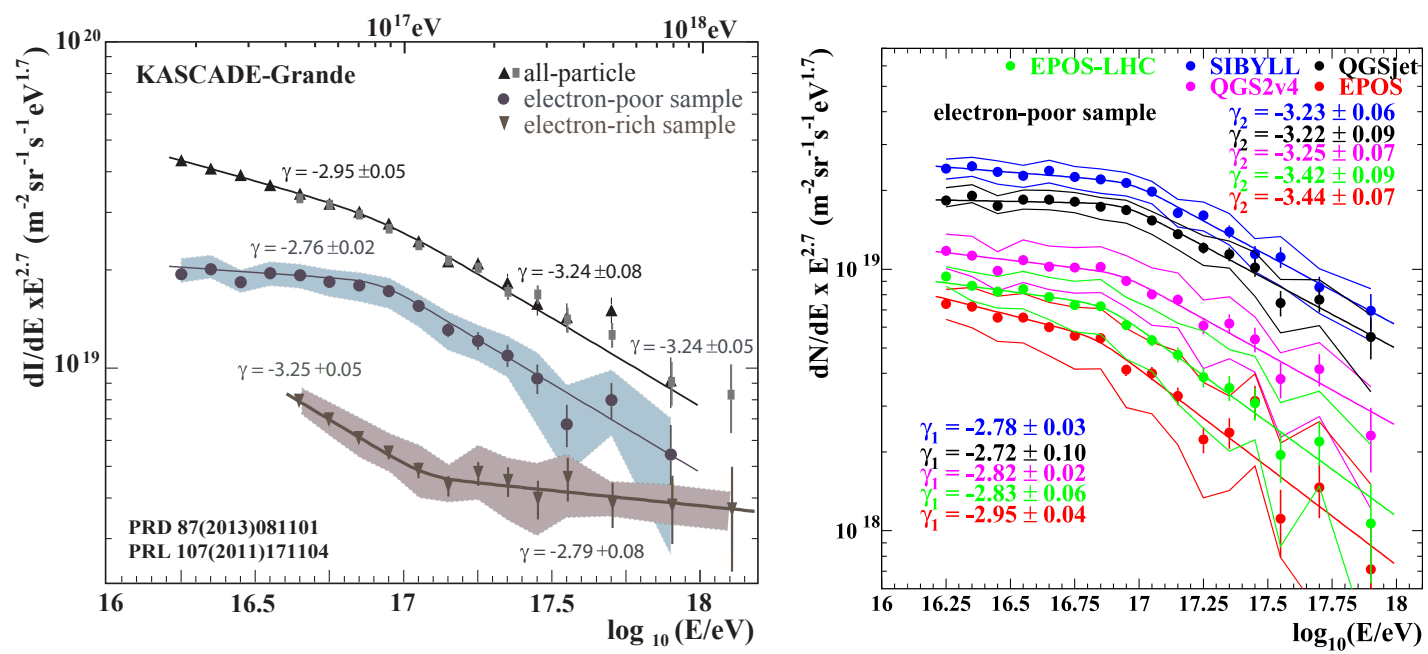

Figure 2: Left: All-particle, electron-poor, and electron rich energy spectra from KASCADE-Grande. Right: Reconstructed energy spectra of the heavy primary component for five hadronic interaction models. The error bars show the statistical uncertainties and the bands the systematic uncertainties; fits on the spectra and resulting slopes before and after the heavy knee are also indicated [12].

\section{Summary of main results}

\subsection{The all-particle energy spectrum}

A composition independent all-particle energy spectrum of cosmic rays was reconstructed in the energy range of $10^{16} \mathrm{eV}$ to $10^{18} \mathrm{eV}$ from the Grande data within a total uncertainty in flux of $10-15 \%$ [5]. The spectrum is in the overlapping region in agreement with the earlier published spectrum by KASCADE [6]. Significant structures are observed in the spectrum (Fig. 1): The justification of the 'knee' at a few times $10^{15} \mathrm{eV}$ is given since many years. But now, there is also a clear evidence that just above $10^{16} \mathrm{eV}$ the spectrum shows a significant 'concave' behavior. Another feature in the spectrum is a small break, i.e. knee-like feature at around $10^{17} \mathrm{eV}$. The latter slope change occurs at an energy where the rigidity dependent, i.e. charge dependent, knee of the iron component would be expected, if the 'knee' is caused by light primaries. The concave part of the spectrum is then a consequence of knee-like features of the spectra of medium masses.

\subsection{Elemental composition of cosmic rays}

Already in 2005 KASCADE could prove [6] that the knee is caused by a decrease of the light mass group of primary particles and not by medium and heavy primary particles. With KASCADEGrande we investigated such individual mass group spectra also at higher primary energies [7, 8]. All the simulations for the described analyses are performed with the air-shower simulation package CORSIKA [9] allowing simulations based on various hadronic interaction models. The application of this methodical approach to shower selection and separation in various mass groups were performed and cross-checked in different ways, where figure 2, left panel, shows the main results: The reconstructed spectrum of the electron-poor events, i.e. the spectrum of heavy primaries, shows a distinct knee-like feature at about $8 \cdot 10^{16} \mathrm{eV}$ with a statistical significance of $3.5 \sigma[7,10]$. 
The analysis was repeated on basis of different hadronic interaction models [11,12]. Despite the fact, that the relative abundance of the heavy particles varies significantly dependent on the model in use, the spectral feature of this 'heavy' knee is visible in all the spectra (Fig. 2, right panel). This fits to a rapidity dependent knee scenario with the results of KASCADE [6], where a reduction of the light component beyond the first knee was observed. In addition, an ankle-like feature was observed in the spectrum of the electron-rich events (Fig. 2, left), i.e. light elements of the primary cosmic rays, at an energy of $10^{17.08 \pm 0.08} \mathrm{eV}$. At this energy, the spectral index changes by $\Delta \gamma=0.46$ with a statistical significance of $5.8 \sigma[8]$.

\subsection{Hadronic interaction models}

A great achievement of KASCADE was the invention of the CORSIKA air-shower simulation tool, which meanwhile is used by all major experiments worldwide. Mainly data of the hadron calorimeter and additional muon counters at the central detector and the muon tunnel were used within KASCADE to iteratively test and improve the various versions of the hadronic interaction models optionally available in CORSIKA (see e.g., $[13,14]$ and references therein).

\subsection{Radio detection of EAS}

Another great achievement was the proof-of-principle that indeed radio detection of extensive air-showers is possible by an array of digital radio antennas [3].

\section{Present activities}

Despite that KASCADE-Grande has terminated the active data acquisition, the collaboration continues the detailed data analysis. A few examples of current works are discussed in the following.

\subsection{KASCADE and KASCADE-Grande combined analysis}

A specific analysis focuses on combining the KASCADE and KASCADE-Grande arrays already at the shower reconstruction. The number of charged particles and the number of muons is the result of a fit to the lateral densities measured by both arrays. Therefore, events located in the Grande array gain additional 252 density measurements and events located in the KASCADE array gain 37 additional measurements compared to the individual fits to the lateral densities. The new 2-dimensional shower size spectrum (Fig. 3, left panel) is then the object of the further analysis. The distribution contains events from a larger fiducial area, are reconstructed with higher accuracy, and cover more than three orders of magnitude in primary energy. The goal of this analysis is to obtain all-particle as well as mass group spectra by one methodically consistent reconstruction procedure [15].

\subsection{Search for primary gamma rays}

Using the KASCADE and KASCADE-Grande arrays, upper limits on the diffuse gamma ray flux are obtained [16] by selecting and studying muon-poor air shower events (fig. 3, right panel). An update on this analysis as well as for the search of point-sources in the gamma enhanced shower sample of KASCADE is presented in this conference [17, 19, 18]. 

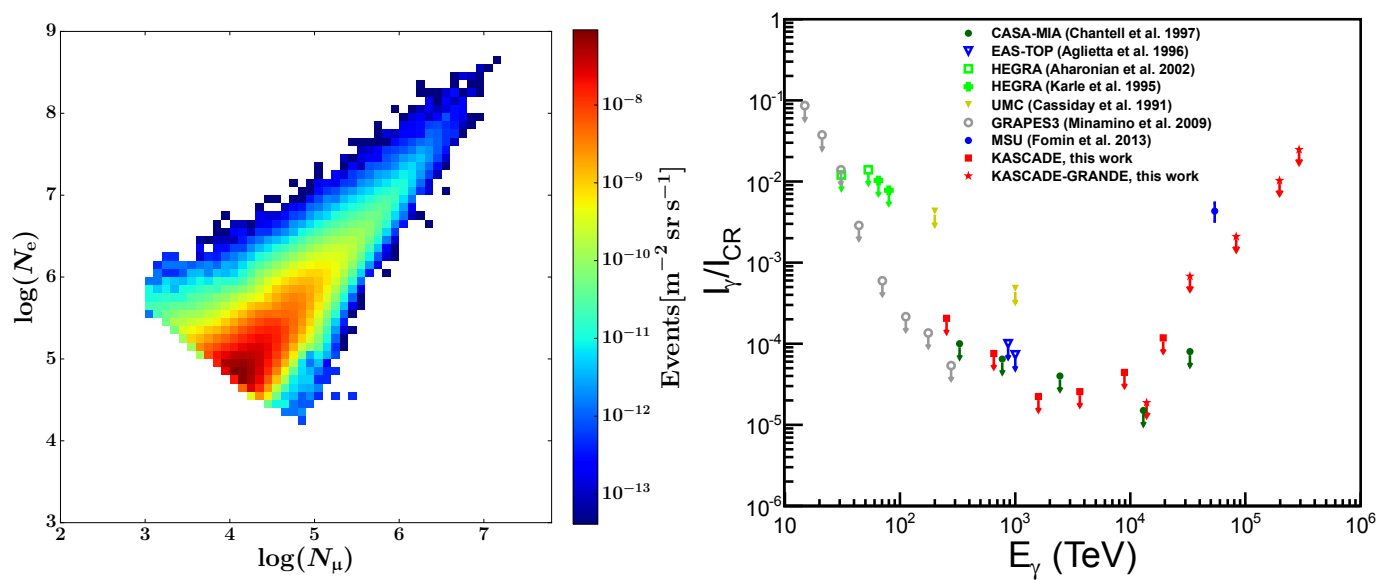

Figure 3: Left: The two-dimensional shower size spectrum for KASCADE-Grande data obtained by a combined shower reconstruction of data from the KASCADE and the Grande arrays [15]. Right: Upper limits on the diffuse gamma ray flux relative to the measured CR flux (See [16]).

\subsection{Muon attenuation length}

Another analysis studies the attenuation of muons and charged particles in the Earths' atmosphere [20]. This is especially interesting for cross-checking hadronic interaction models. The number of muons measured for a certain flux is decreasing with increasing zenith angle. Therefore, the attenuation of muons in the atmosphere can be obtained (Fig. 4, left panel) where it is clear that none of the four models studied describes the observed attenuation length. However, the effect of this deficiency to the standard energy and composition reconstruction at KASCADE-Grande is small and determined as less than $10 \%$, included in the estimation of the systematic uncertainties.

\subsection{The S(500) parameter}

Related to the uncertainties in estimating the energy spectra there is an analysis ongoing that
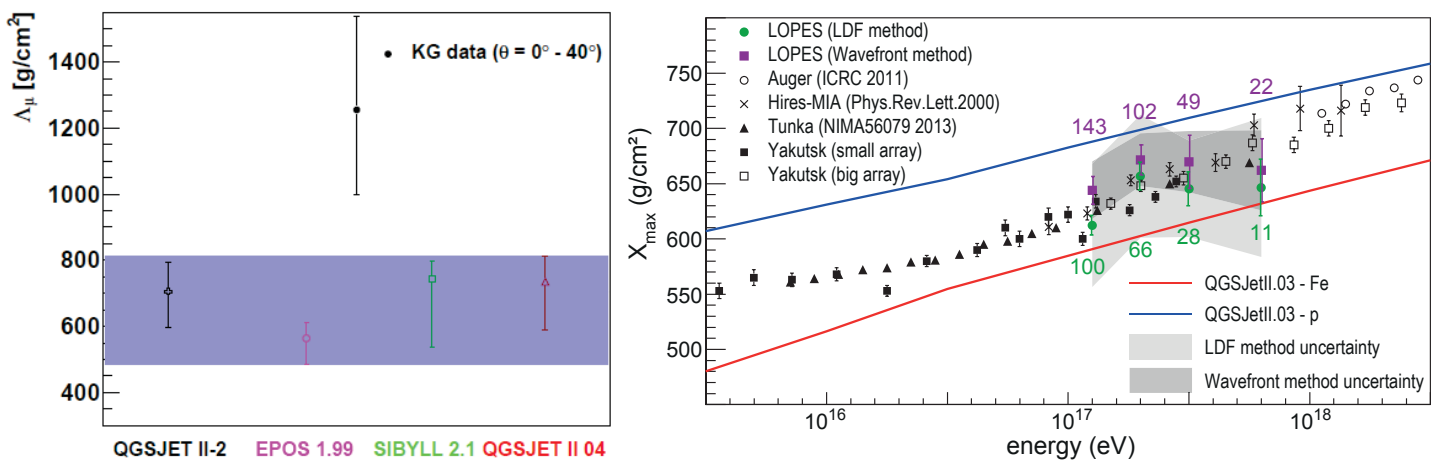

Figure 4: Left: Muon attenuation lenghts for KASCADE-Grande in comparison with those obtained by detailed Monte-Carlo simulations based on various hadronic interaction models [20]. Right: Measurements of different experiments of the mean atmospheric depth of the shower maximum vs. energy. The results for LOPES are based on two independent methods [23]. The indicated number of events per bin deviate between both methods due to different quality cuts. 

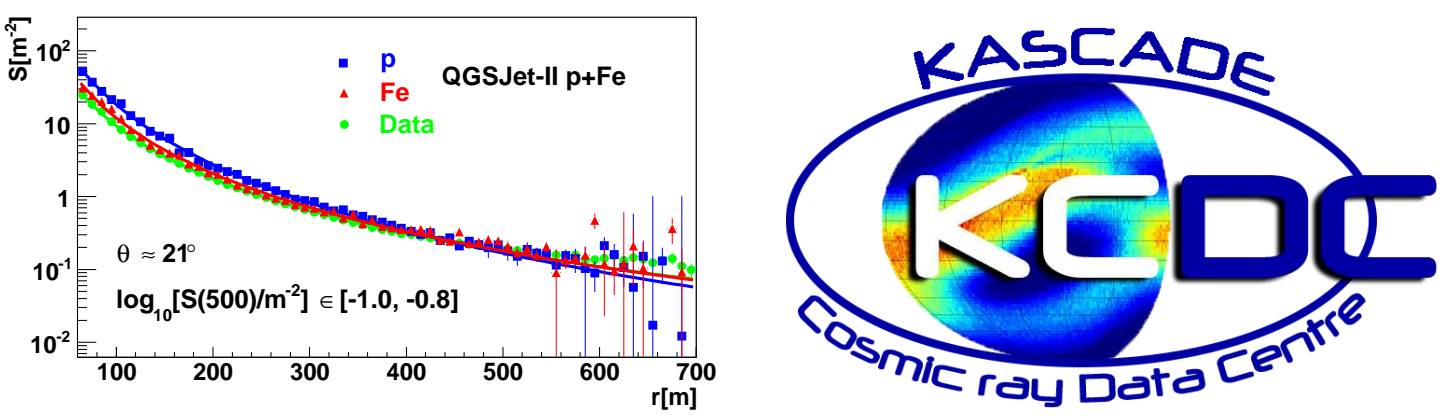

Figure 5: Left: Averaged lateral charged particle density distributions for simulations and experimental data for the same value range of S(500). Right: The KCDC logo.

investigates the use of another observable, namely $S(500)$, for the reconstruction of the primary energy [21, 22]. It is based on the assumption that, for the KASCADE-Grande array, the lateral density distributions for all elements cross at a distance of around 500 meters from the shower-core. The density at this distance is, therefore, independent of the mass of the primary particle and its value is sensitive to the primary energy. However, it was found that there is a systematic shift in the reconstructed energy (at data) where we could assign an insufficient shape of the lateral distribution in the simulations as reason for the discrepancy (Fig. 5, left panel).

\subsection{Radio detection within the KASCADE facility}

After the proof-of-principle of this detection technology in 2005 [3], first analyses of the primary UHECR parameters, like energy and mass are performed. The right panel of figure 4 shows two examples, where the LOPES experiment (antennas sensitive to the $\mathrm{MHz}$ frequency range) applied two different methods (using the lateral distribution of the radio signal, and the shape of the wavefront, respectively) to determine the shower maximum [23]. This is the first measurement of the shower maximum by the radio detection technique. The results are very promising for the future, despite the fact that at LOPES the systematic uncertainties are very large due to the humanmade noise at the industrial environment of KASCADE-Grande.

Within KASCADE-Grande by the CROME experiment also a first systematic study of the $\mathrm{GHz}$ emission in EAS showers was possible as for more than 30 events unambiguously a radiation in this frequency range could be observed [24].

\subsection{The KASCADE Cosmic-ray Data Center: KCDC}

The KASCADE facility operated several large-area detectors for the measurement of EAS for more than 20 years. The aim of KCDC is the installation and establishment of a public data centre for high-energy astroparticle physics. In the research field of astroparticle physics, such a data release is a novelty, whereas the data publication in astronomy has been established for a long time. The first goal of KCDC (Fig. 5, right panel) was to make the data from the KASCADE experiment available to the community, where the initial platform to external users is already released [25]. Interested colleagues are invited to visit KCDC under https: / / kcdc.ikp. kit. edu. 


\section{KASCADE timeline}

- 53 collaborative papers in reviewed journals (8 still in queue, short author list papers not included)

- $55 \mathrm{PhD}$ thesis

- $86 \mathrm{diploma/master} \mathrm{thesis}$

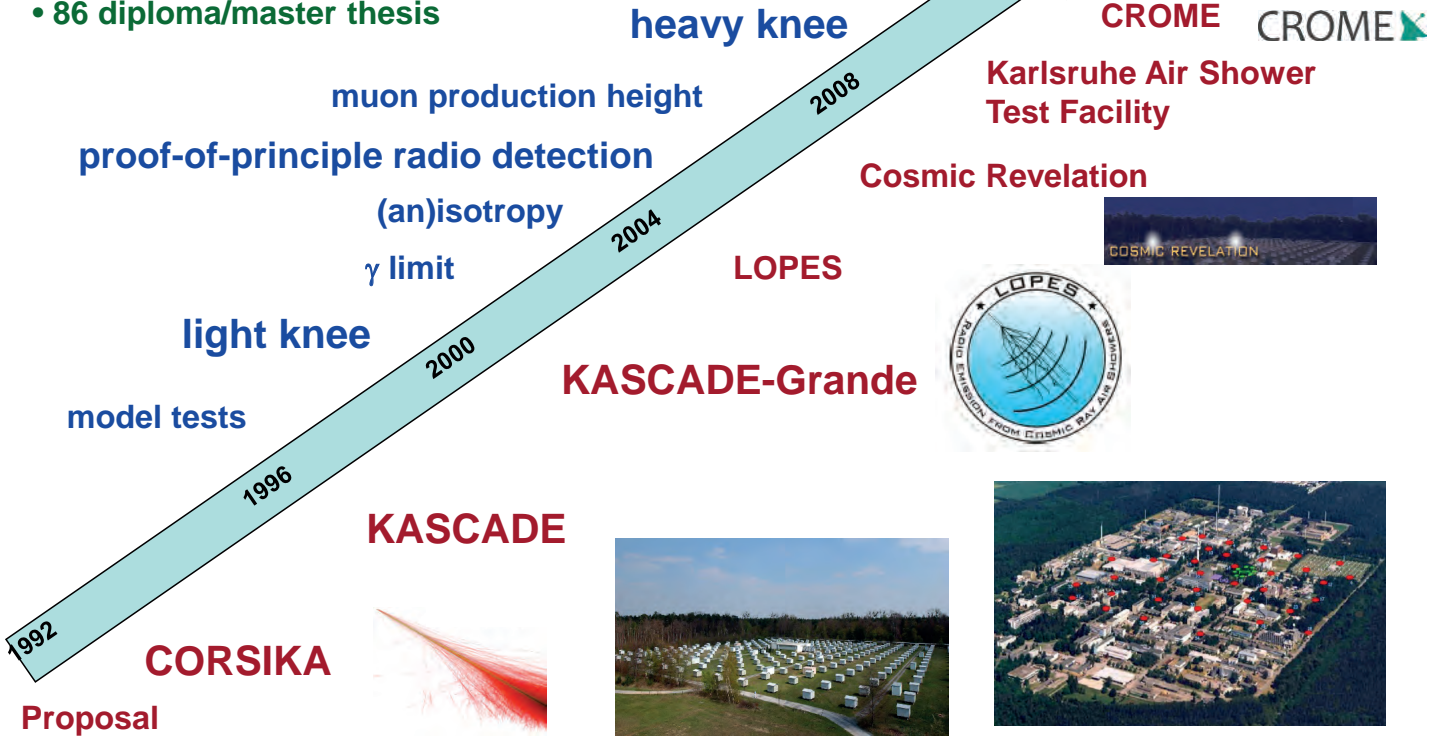

Figure 6: Timeline of the KASCADE(-Grande) facility. Shown are below the arrow of time in red the various hardware related parts of the facility and above the arrow in blue some highlights of physics results. 'Cosmic Revelation' was an art \& science project at KASCADE in 2009 [26].

\section{Summary}

In summary, after separating the KASCADE-Grande measured events into light and heavy components, knee-like features are identified in the spectrum of the light component by KASCADE and of the heavy component by Grande, as well as an ankle-like feature is observed in the spectrum of the light component. Whereas the 'heavy-knee' occurs at an energy where the rigidity dependent knee of the iron component is expected, the 'light-ankle' might indicate an early transition from galactic to extragalactic origin of cosmic rays. The KASCADE-Grande collaboration has started to provide the entire data set to the public within the project KCDC, the KASCADE Cosmic Ray Data Center.

Lessons learnt from 25-years KASCADE facility In the following there are a few arguments listed which probably made KASCADE to the succesful experimental set-up as it happened. The Figure 6 illustrates the timeline and some highlights of 20 years KASCADE(-Grande) facility.

- It is essential to provide spectra of individual mass groups and not a mean logarithmic mass, as for the latter one the ambiguities in spectral shapes are too large.

- One (still) needs multi-parameter EAS measurements to validate the hadronic interaction models and also to perform complementary particle physics to the man-made accelerators. 
- For astrophysical interpretations a multi-messenger detection (at least for charged cosmic rays and gammas) is advantageous.

- It is important to deliver high statistics in a large energy range, as probably only composition dependent anisotropy studies will give new insights.

- A facility should provide enough room for R\&D studies for the development of future and/or improved detection technologies.

- In present time a coherent outreach and public data access policy gets more and more important.

Acknowledgments The authors would like to thank the members of the engineering and technical staff of the KASCADEGrande collaboration, who contributed to the success of the experiment. The KASCADE-Grande experiment is supported in Germany by the BMBF and by the 'Helmholtz Alliance for Astroparticle Physics - HAP' funded by the Initiative and Networking Fund of the Helmholtz Association, by the MIUR and INAF of Italy, the Polish Ministry of Science and Higher Education, and the Romanian Authority for Scientific Research UEFISCDI (PNII-IDEI grants 271/2011 and 17/2011). J.C.A.V. acknowledges the partial support of CONACyT.

\section{References}

[1] Antoni T et al. - KASCADE, NIM A $\mathbf{5 1 3} 490$ (2003)

[2] Apel W D et al. - KASCADE-Grande, NIM A 620202 (2010)

[3] H. Falcke et al. - LOPES, Nature 435313 (2005)

[4] R. Smida et al. - CROME, EPJ Web Conf. 5308010 (2013)

[5] W.-D. Apel et al. - KASCADE-Grande, Astropart. Phys. 36183 (2012)

[6] T. Antoni et al. - KASCADE, Astropart. Phys. 241 (2005)

[7] W.-D. Apel et al. - KASCADE-Grande, PRL 107171104 (2011)

[8] W.-D. Apel et al. - KASCADE-Grande, $P R D 87$ 081101(R) (2013)

[9] D. Heck et al., report Forschungszentrum Karlsruhe, FZKA 6019 (1998)

[10] W.-D. Apel et al. - KASCADE-Grande, Astropart. Phys. 4754 (2013)

[11] W.-D. Apel et al. - KASCADE-Grande, Adv. Space Res. 531456 (2014)

[12] M. Bertaina et al. - KASCADE-Grande, ICRC 2015, The Netherlands, PoS(ICRC2015)359

[13] T. Antoni et al. - KASCADE, J. Phys.G 36035201 (2009)

[14] W.-D. Apel et al. - KASCADE-Grande, Astrop. Phys. 6555 (2015)

[15] S. Schoo et al. - KASCADE-Grande, ICRC 2015, The Netherlands, PoS(ICRC2015)263

[16] D. Kang et al. - KASCADE-Grande, Proc. ECRS, Kiel Germany (2014) to be published (2015)

[17] D. Kang et al. - KASCADE-Grande, ICRC 2015, The Netherlands, PoS(ICRC2015)785

[18] Z. Feng et al. - KASCADE, ICRC 2015, The Netherlands, PoS(ICRC2015)823

[19] D. Kang, Z. Feng et al. - KASCADE, ICRC 2015, The Netherlands, PoS(ICRC2015)788

[20] J.C. Arteaga et al. - KASCADE-Grande, ICRC 2015, The Netherlands, PoS(ICRC2015)314

[21] G. Toma et al. - KASCADE-Grande, ICRC 2015, The Netherlands, PoS(ICRC2015)301

[22] A. Gherghel-Lascu et al. - KASCADE-Grande, ICRC 2015, The Netherlands, PoS(ICRC2015)302

[23] F.G. Schröder et al. - LOPES, Proc. ECRS, Kiel Germany (2014) to be published (2015)

[24] R. Smida et al. - CROME, PRL 113221101 (2014)

[25] S. Schoo et al. - KASCADE-Grande, ICRC 2015, The Netherlands, PoS(ICRC2015)262

[26] T.O. Roth and A. Haungs, Transactions, Leonardo 423 (2009) 